\title{
A unique case of benign pleomorphic adenoma metastasizing to the thyroid gland and liver
}

\author{
Mufaddal Moonim ${ }^{1}$, Katherine Wiles ${ }^{1}$, Alia Nasir ${ }^{2}$, Ricard Simo ${ }^{1}$ \\ 1. Department of Otorhinolaryngology Head and Neck Surgery, Guy's Hospital, Great Maze Pond Road, London SE1 9RT, \\ United Kingdom. 2. Department of Histopathology, St Thomas's Hospital, Westminster Bridge Road, London SE1 7EH, \\ United Kingdom.
}

Correspondence: Katherine Wiles. Address: Department of Head and Neck Surgery, Guy's Hospital, Great Maze Pond Road, London SE1 9RT, United Kingdom. E-mail: katherine.wiles@kcl.ac.uk

Received: August 5, 2015

DOI : $10.5430 /$ crcp.v2n4p74

Online Published: September 22, 2015

\section{Abstracts}

A recurrent benign metastasizing pleomorphic salivary adenoma (PSA) is extremely rare occurring in less than $1 \%$ of patients. There are approximately 50 cases that have been reported in the literature but none with metastases to the thyroid gland. We report a case of a 68-year-old lady who was initially investigated for a space-occupying lesion in the liver but was found to have metastases in the thyroid and liver as well as recurrence of the parotid primary. The clinical findings, management, and a review of the current literature are reported. Metastases from PSAs are extraordinary. We describe the first reported metastasis from the parotid to the thyroid gland. In this case, we report an excellent outcome post-operatively and argue for the benefit of long-term follow-up.

\section{Key words}

Benign pleomorphic adenoma, Salivary gland, Metastasis, Thyroid, Liver

\section{Introduction}

Recurrent benign metastasizing pleomorphic salivary adenoma (PSA) is extremely rare, occurring in less than $1 \%$ of patients with PSA. However, when PSAs recur, they are associated with poorer outcomes and prognosis ${ }^{[1]}$. Pleomorphic adenoma in the thyroid gland has been described previously: one case in the right thyroid lobe ${ }^{[2]}$ and the other as an incidental midline isthmic thyroid nodule ${ }^{[3]}$. Both of these were arising as a primary tumor in the thyroid with no evidence of a salivary gland primary.

We report a case of PSA of the parotid gland, which recurred locally in the parotid gland with synchronous metastases in the thyroid and liver. This finding constitutes the first reported case of benign metastases of a PSA from the parotid to the thyroid gland and liver in the same patient. The patient was treated with surgical excision of the recurrent tumor and metastases. The patient is symptom-free without recurrence at 2 years from completion of treatment.

\section{Case report}

A 68-year-old female presented to the hepatology team with idiopathic thrombocytopenic purpura (ITP). The patient did not have any other significant past medical history except that she underwent the excision of a PSA of the right parotid 
gland 52 and 35 years ago. Details of the original surgery were not available. The PSA recurred again in 1995 and was left untreated at the patient's request. As part of her diagnostic work-up for ITP in 2013, a Positron emission tomographycomputed tomography (PET-CT) demonstrated uptake in the left lobe of the liver. A left segment hepatectomy was advised and performed, and the lesion fully excised. Histopathological analysis demonstrated a benign PSA.

The patient was subsequently referred to the senior author for further management. On examination she was found to have a $3 \mathrm{~cm} \times 3 \mathrm{~cm}$ smooth, but firm, mass in the right tail of the parotid gland with no palpable lymphadenopathy (see Figure 1a). Ultrasound scan (US) revealed a hypoechoic $1.7 \mathrm{~cm} \times 1.2 \mathrm{~cm} \times 2 \mathrm{~cm}$ lesions in the right parotid, along with a smaller $7 \mathrm{~mm} \times 4 \mathrm{~mm} \times 5 \mathrm{~mm}$ satellite lesion adjacent to the primary lesion. The scan of the neck also identified a $1.5 \mathrm{~cm}$ $\times 0.9 \mathrm{~cm} \times 1.2 \mathrm{~cm}$ hypoechoic lesions with an irregular, almost scalloped outline in the right lobe of the thyroid. An ultrasound guided fine needle aspiration (USS-FNA) of the parotid thyroid lesions was performed. Cytological analysis, demonstrated abundant myxofibrillary matrix admixed with singly scattered and small clusters of bland plasmacytoid cells in the parotid lesions and the thyroid consistent with benign PSA of the right parotid gland with metastases to the right thyroid.
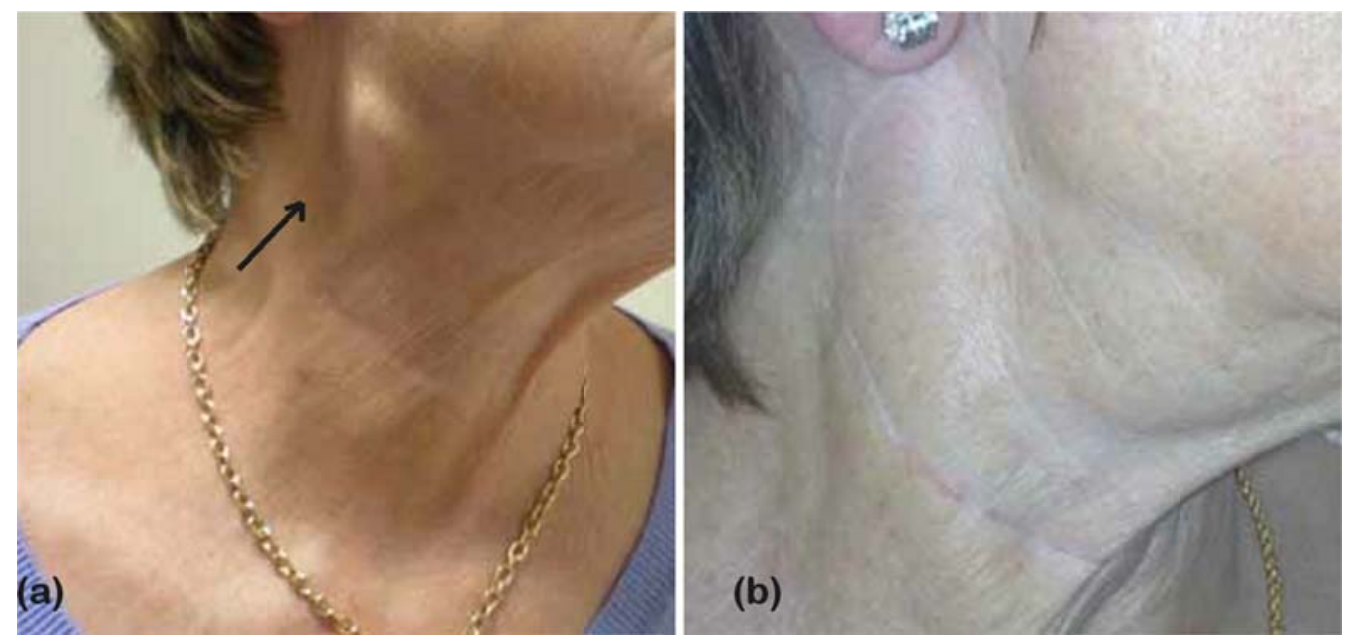

Figure 1. View of the right lateral neck (a) Pre-operatively and (b) Post-operatively (at 18 months follow-up)

Figure 2. Post-surgical excision of the right parotid gland and right thyroid lobe

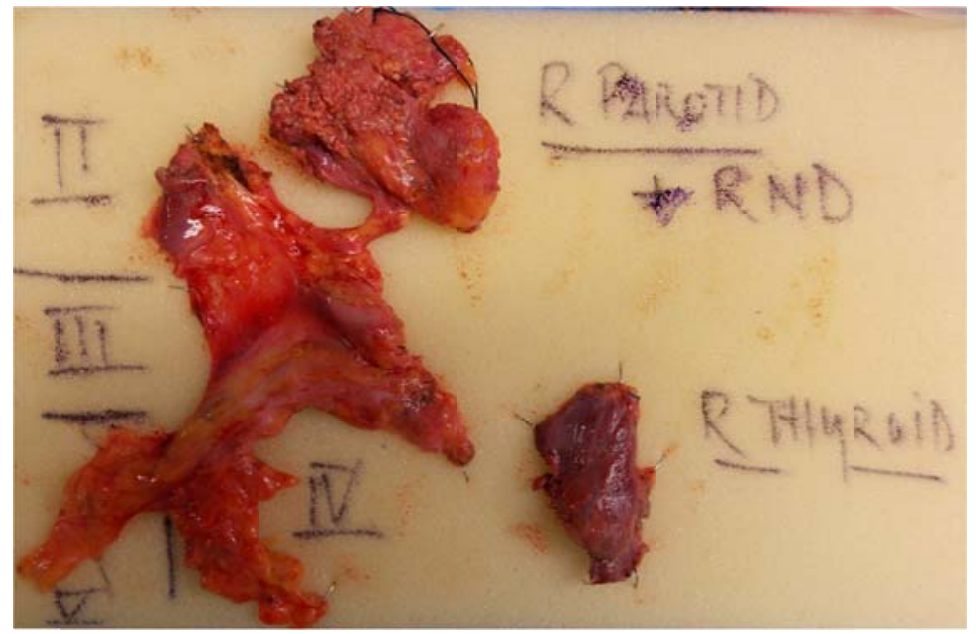

The patient was discussed in the head and neck and thyroid multidisciplinary team meeting. A revision, total conservative right parotidectomy with a right selective neck dissection with a right thyroid lobectomy was recommended. The procedure was done via an extended cervico-facial incision. The facial, vagus, accessory, hypoglossal and recurrent 
laryngeal nerves were identified and preserved, and the dissection was done in continuity with adequate margins (see Figure 2). She underwent an uneventful recovery and was discharged home three days post-operatively.
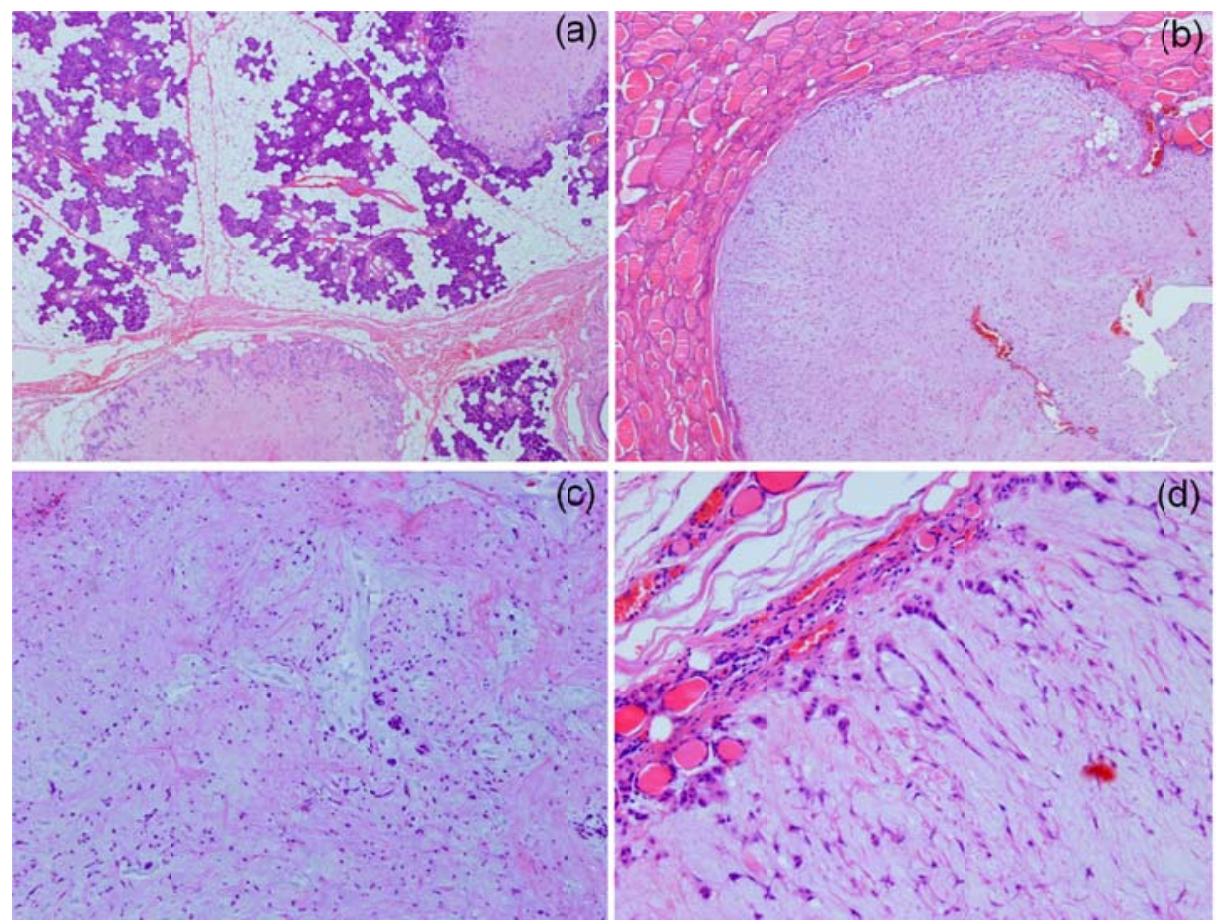

Figure 3. Microscopic lesions on H\&E staining included (a) microscopic pleomorphic adenomas within the parotid gland (magnification 100×), and (b) a well-circumscribed nodule within the thyroid (magnification 100×) demonstrating, (c) myxoid stroma with stellate cells (magnification 200×), and (d) epithelial cells at the periphery (magnification 200×).
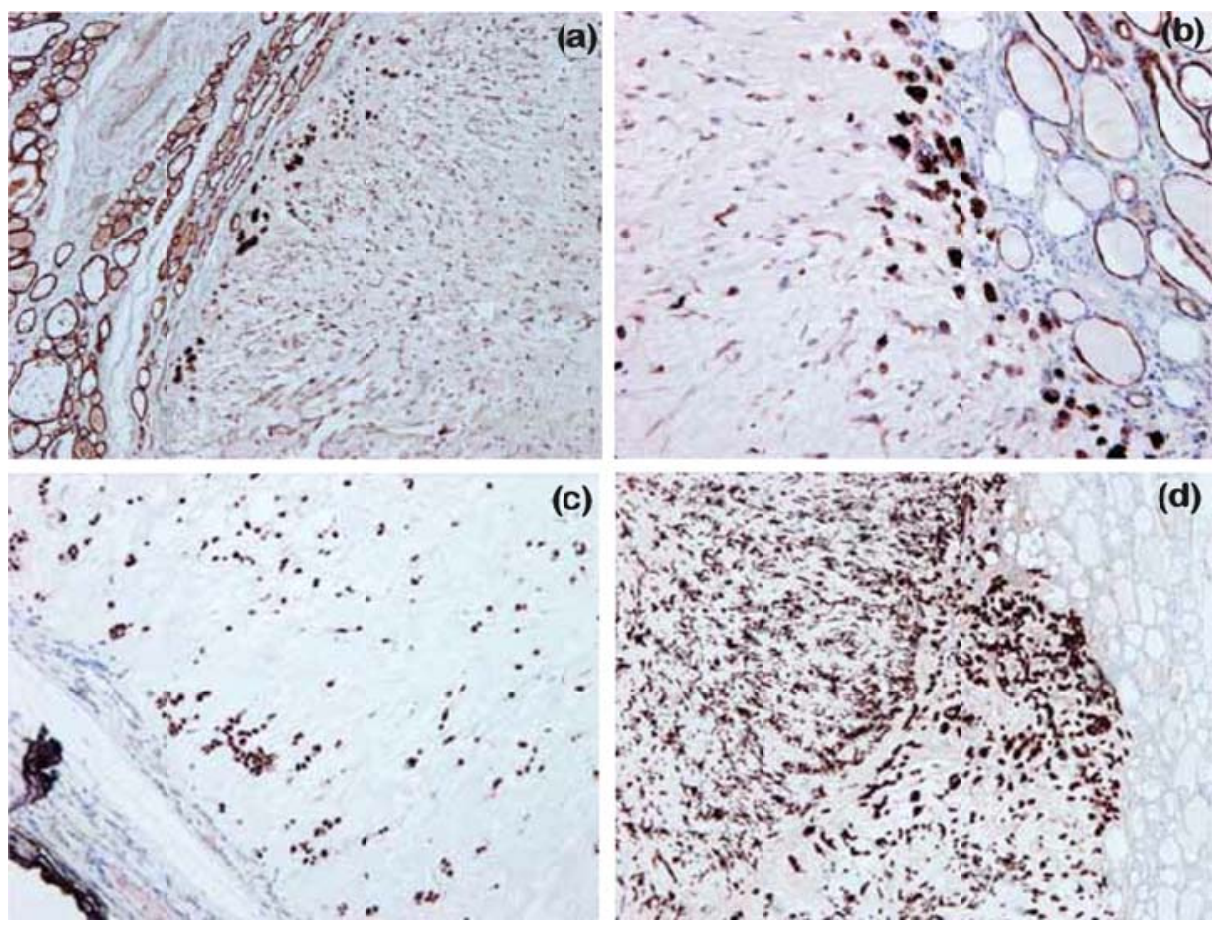

Figure 4. Excised thyroid myoepthelial cells are (a) CK7+, (b) MNF-116+, (c) p63+, and (d) S100+ (Magnification 100×) 
Histopathological analysis of the parotid revealed a well-circumscribed nodule with pushing margins consistent with the hypocellular myxoid variant of PSA. There were six additional microscopic pleomorphic adenomas scattered across the parotid (see Figure 3a). Three were in the accessory lobe and three in the main parotid gland. 35 lymph nodes were dissected from the neck with none demonstrating any features of pleomorphic adenoma. There was no evidence of carcinoma ex pleomorphic adenoma.

The right thyroid lobe also showed a deposit of metastatic PSA that had been completely excised (see Figure 3b-d). This diagnosis was confirmed by immunohistochemistry: the epithelial cells were strongly positive with MNF116 and CK7, while myoepithelial cells expressed both weakly (see Figure 3a and 3b). Myoepithelial cells were also S100(+) and p63(+) (see Figure 3c and 3d). Both cell types were negative for CK20, CK14, SMA, CD10, thyroglobulin and calcitonin.

The patient's case was subsequently discussed in the Head and Neck and Thyroid MDT and no further adjuvant treatment was recommended. Two years following completion of treatment, the patient is asymptomatic with no evidence of disease at the primary site or the thyroid (see Figure 1b). She will be followed up long term with half yearly reviews with US and MRI. Her follow-up by the liver team will comprise yearly CT for the first three years followed by MRI yearly up to five years. Thereafter, she will be offered yearly US scans.

\section{Discussion}

Metastatic recurrent benign pleomorphic adenoma is extremely rare. There are 51 reported cases of benign metastasizing PSA and we report the first case of benign PSA metastasizing to the thyroid and liver at the same time.

Metastasizing PSA are commonly preceded by multiple (at least two to three) local recurrences before the development of metastatic foci. $40 \%-73 \%$ of recurrent tumours are multifocal as compared to $0.14 \%-0.6 \%$ in primary untreated pleomorphic adenoma ${ }^{[1]}$. Renehan et al. have reported bone as the most common site for metastases (45\%) followed by the head and neck (43\%) and lung (36\%) ${ }^{[4]}$. Metastatic deposits have also been discovered in regional lymph nodes, the oral cavity, pharynx, skin, liver, retroperitoneum, kidney, calvarium, and central nervous system ${ }^{[5]}$.

The lack of atypical features at the metastatic sites is consistent with the observation that predictable morphological features of metastasizing tumours such as cellular atypia are not known to occur in metastatic benign PSA ${ }^{[6]}$. Czader et al. suggest that metastasizing PSA is an intermediate step between a benign primary and carcinoma ex-PSA whereby the accumulation of genetic mutations increases the potency of the tumour ${ }^{[7]}$. Genelhu et al. note that p63 is a potent marker of myoepithelial differentiation that is present in PSA but absent in carcinoma ex pleomorphic adenoma ${ }^{[8]}$. S100 is another marker that has variable expression in carcinoma ex-PSA but is always present in benign PSA ${ }^{[9]}$. In our analysis, the metastasizing PSA was still p63 and S100 positive, suggesting that loss of differentiation had not occurred.

The clinical presentation of recurrent PSA depends on the site affected. In this the metastasis to the thyroid was clinically silent. The majority of patients with recurrent PSA present with a slowly enlarging painless mass in the parotid bed ${ }^{[10]}$. The patient in this case had noted a recurrent PSA but, as this was asymptomatic, she did not seek any medical attention. The resulting delay between the recurrence and subsequent clinical symptoms was 17 years.

In patients with recurrent PSA, a vigilant approach to extra-parotid symptoms has been advocated due to the risk of malignant transformation. The category of potential malignant mixed tumours of the salivary gland includes metastasizing pleomorphic adenoma, carcinoma ex-PSA, and carcinosarcoma. Carcinoma ex-PSA is an epithelial malignancy that is derived from a pleomorphic adenoma. It can be further subdivided into intracapsular (non-invasive, in-situ), minimally invasive, and invasive carcinomas. The risk of transformation to carcinoma ex-PSA has been estimated at an approximate rate of $1 \%$ per year but may be as high as $25 \%{ }^{[11]}$. Carcinosarcoma is a true malignant mixed tumour containing both 
epithelial and mesenchymal components. Carcionsarcoma is a highly aggressive tumour that, like carcinoma ex-PSA, carries a poor 5-year survival ${ }^{[12,13]}$.

The cause of PSA metastasis is currently unknown. It has been hypothesized that either inadequate surgical procedures, such as incomplete surgery, or surgical manipulation may cause tumour cell dislodgement and vascular penetration with subsequent haematogenous spread ${ }^{[5]}$. Haematogenous metastasis is more prevalent than metastasis to regional lymph nodes ${ }^{[14]}$. However, a lymphatic metastatic route has also been reported ${ }^{[15]}$. Here, the presence of deposits in the liver and thyroid as well as the lack of lymphatic involvement suggests a haematogenous route of spread.

The evaluation and diagnosis of recurrent PSA is often difficult due to fibrotic changes caused by previous surgery. USS-FNA is the mainstay of investigation as it can identify recurrence and guides surgical treatment for recurrent PSA. Further pathological analysis can also determine whether there are any metastatic features such as cellular atypia and mitotic features. Computerized axial tomograpy (CT) and Magnetic resonance imaging (MRI) are both excellent imaging tools also used to determine features of possible malignant transformation. Moonis et al. argue for the increasing role of MRI in order to rule out malignant metastasis in cases where the PSA is not being operated on or to check for sub-clinical recurrence. MRI not only has excellent spatial resolution, but it can also determine involvement of the facial nerve, perineural areas, bone, and meninges. Furthermore, MRI confers excellent visibility of the neoplasm on T2 weighted images ${ }^{[16]}$.

The management of patients with recurrent and metastatic PSA is challenging. Although most recurrent PSAs are clinically asymptomatic, as already noted, these patients are at an increased risk of malignant transformation. PSAs are contained within a pseudocapsule but have finger-like projections ${ }^{[17]}$. Therefore, total conservative parotidectomy with adequate margins and preservation of the facial nerve is the treatment of choice, and the use of intra-operative facial nerve monitoring is advisable ${ }^{[18]}$. Revision surgery of the parotid can be extremely difficult due to the presence of fibrosis and should be reserved for experienced surgical teams dealing regularly with this cohort of patients.

For solitary metastases, metastasectomy is advised whenever possible. It confers a significant survival advantage in univariate log-rank analysis over non-operative treatment, with five-year disease-free survivals of $58 \%$ and $50 \%$, respectively ${ }^{[6]}$. A selective or supra-selective neck dissection is appropriate to achieve adequate margins. There is no available literature reporting metastasis of recurrent PSA in the thyroid gland ${ }^{[19]}$. As there was only one metastasis in the thyroid gland clinically and radiologically, a thyroid lobectomy rather than a total thyroidectomy was performed.

The use of postoperative radiotherapy for recurrent PSA has been widely discussed with several studies indicating no benefit to the use of radiotherapy after uncomplicated parotidectomy for single, localized recurrences ${ }^{[20,21]}$. There is evidence to suggest that post-operative radiotherapy helps local control and should be employed in multi-centric recurrences or if there is doubt of complete excision ${ }^{[18]}$. Treating teams may also take into account age, treatment morbidity, facial nerve involvement, and contraindications to further surgery when considering radiotherapy ${ }^{[20]}$. In the case we report, the patient did not undergo radiotherapy and is clinically disease free at 2 years follow-up.

The development of metastases within the first 10 years of diagnosis or at multiple sites are independent predictors of worse survival ${ }^{[15]}$. Long-term follow-up is therefore necessary after treatment of a metastatic lesion considering the demonstrated delay in presentation of subsequent pathology ${ }^{[5]}$. Therefore, the patient is undergoing half-yearly follow-up with USS and MRI scan as well as review under the liver team.

\section{References}

[1] Akiba J, et al. A case of metastasizing pleomorphic adenoma in the maxillary bone appearing twenty years after initial resection of pleomorphic adenoma of the hard palate. Pathology international. 2013; 63(9): 463-468. http://dx.doi.org/10.1111/pin.12087 
[2] Lange M. Pleomorphic adenoma of the thyroid containing salivary gland cells with pseudocartilage and myoepithelial cells. International surgery. 1974; 59(3): 178. PMid: 4363238.

[3] Levy GH, et al. Pleomorphic adenoma arising in an incidental midline isthmic thyroid nodule: a case report and review of the literature. Human pathology. 2012; 43(1): 134-137. PMid: 21733552. http://dx.doi.org/10.1016/j.humpath.2011.02.025

[4] Renehan A, et al. Long, Äêterm follow, Äêup of over 1000 patients with salivary gland tumours treated in a single centre. British journal of surgery. 1996; 83(12): 1750-1754. PMid: 9038559. http://dx.doi.org/10.1002/bjs.1800831228

[5] Manucha V, OB Ioffe. Metastasizing pleomorphic adenoma of the salivary gland. Archives of pathology \& laboratory medicine. 2008; 132(9): 1445-1447. PMid: 18788859.

[6] Nouraei S, et al. Metastasizing pleomorphic salivary adenoma. Archives of Otolaryngology, ÄìHead \& Neck Surgery. 2006; 132(7): 788-793. PMid: 16847191. http://dx.doi.org/10.1001/archotol.132.7.788

[7] Czader M, et al. Metastasizing mixed tumor of the parotid: initial presentation as a solitary kidney tumor and ultimate carcinomatous transformation at the primary site. The American journal of surgical pathology. 2000; 24(8): 1159-1164. PMid: 10935658. http://dx.doi.org/10.1097/00000478-200008000-00015

[8] Genelhu MC, et al. Immunolocalization of $\beta$-catenin in pleomorphic adenomas and carcinomas ex-pleomorphic adenomas of salivary glands. Applied Immunohistochemistry \& Molecular Morphology. 2007; 15(3): 273-278. PMid: 17721271. http://dx.doi.org/10.1097/01.pai.0000213123.04215.95

[9] Zarbo RJ, et al. Salivary gland basal cell and canalicular adenomas: immunohistochemical demonstration of myoepithelial cell participation and morphogenetic considerations. Archives of pathology \& laboratory medicine. 2000; 124(3): 401-405. PMid: 10705394.

[10] Leonetti JP, et al. Recurrent pleomorphic adenoma of the parotid gland. Otolaryngology-Head and Neck Surgery. 2005; 133(3): 319-322. PMid: 16143173. http://dx.doi.org/10.1016/j.otohns.2005.04.008

[11] Dhillon M, et al. Carcinoma Ex Pleomorphic Adenoma of Parotid Gland with Hepatic Metastasis: Clinic-Radiological Case Report. 2014.

[12] Wahlberg P, et al. Carcinoma of the parotid and submandibular glands-a study of survival in 2465 patients. Oral oncology. 2002; 38(7): 706-713. http://dx.doi.org/10.1016/S1368-8375(02)00007-6

[13] Tortoledo ME, Luna MA, Batsakis JG. Carcinomas ex pleomorphic adenoma and malignant mixed tumors: histomorphologic indexes. Archives of otolaryngology. 1984; 110(3): 172-176. PMid: 6322732. http://dx.doi.org/10.1001/archotol.1984.00800290036008

[14] Chen KT. Metastasizing pleomorphic adenoma of the salivary gland. Cancer. 1978; 42(5): 2407-2411. http://dx.doi.org/10.1002/1097-0142(197811)42:5<2407::AID-CNCR2820420542>3.0.CO;2-Y

[15] Wenig BM, et al. Metastasizing mixed tumor of salivary glands: a clinicopathologic and flow cytometric analysis. The American journal of surgical pathology. 1992; 16(9): 845-848. PMid: 1384375. http://dx.doi.org/10.1097/00000478-199209000-00003

[16] Moonis G, et al. Imaging characteristics of recurrent pleomorphic adenoma of the parotid gland. American Journal of Neuroradiology. 2007; 28(8): 1532-1536. PMid: 17846206. http://dx.doi.org/10.3174/ajnr.A0598

[17] Donovan DT, Conley JJ. Capsular significance in parotid tumor surgery: reality and myths of lateral lobectomy. The Laryngoscope. 1984; 94(3): 324-329. PMid: 6321863. http://dx.doi.org/10.1288/00005537-198403000-00006

[18] Witt RL, et al. Etiology and management of recurrent parotid pleomorphic adenoma. The Laryngoscope. 2014.

[19] Philouze P, et al. Massive Multinodular Head and Neck Recurrence of Parotid Gland Pleomorphic Adenoma: A Case Report. Case reports in otolaryngology. 2014.

[20] McGurk M, Combes J. Controversies in the management of salivary gland disease. Oxford University Press. 2012. http://dx.doi.org/10.1093/med/9780199578207.001.0001

[21] Robertson B, et al. Pleomorphic adenomas: Post-operative radiotherapy is unnecessary following primary incomplete excision: A retrospective review. Journal of Plastic, Reconstructive \& Aesthetic Surgery. 2014. 